\title{
\begin{tabular}{l|l} 
pcori $).$ & PATIENT-CENTERED OUTCOMES RESEARCH INSTITUTE \\
RESEARCH SUMMARY
\end{tabular}
}

\section{Using a Bayesian Approach to Predict Patients' Health and Response to Treatment}

Principal investigator

Scott Zeger, PhD
Organization

Johns Hopkins University

\section{What was the project about?}

When choosing a treatment, doctors often look at research results that show how well the treatment worked in large groups of people. But many factors can affect how well a treatment works for an individual patient. These factors may include the patient's sex, age, other health problems, or how they responded to treatments in the past. Some patient data sources, such as electronic health records, have this information. But existing statistical methods may not use these data well. For example, existing methods may not be able to take advantage of data that include measurements of a patient's health from more than one point in time.

For this project, the research team developed new methods to analyze data that includes measurements of a patient's health from different points in time. To develop the new methods, the team used a Bayesian approach. Bayesian approaches include findings from previous studies in the analysis, which can make results more accurate.

\section{What did the research team do?}

The research team used data on a patient's own health and on how well a treatment worked for others to create the new methods. To help researchers use the new methods, the team created a computer program. Finally, the team used three studies to adapt the methods to predict health changes and responses to treatment for patients having one of three different health problems.
Patients, doctors, and a health plan administrative leader provided input to build and refine the methods. A group of researchers helped to create the computer program.

\section{What were the results?}

The methods predicted aspects of a patient's health and changes in health over time. In the first study, the new methods helped researchers find out whether a child's pneumonia was caused by a virus or bacteria. In the second study, the team used the new methods to see if active monitoring or surgery would work better for a patient with prostate cancer. In the third study, the new methods helped to predict a patient's mental health symptoms over time.

\section{What were the limits of the project?}

The research team tested the new methods with three health problems; the methods might work differently for other health problems.

Future research could test the methods with other examples. Studies could also look at whether using the methods for treatment decisions can help improve patients' health.

\section{How can people use the results?}

Researchers can use the methods to help predict changes in a patient's health and how well a treatment will work.

To learn more about this project, visit www.pcori.org/Zeger319. 